\title{
DRY AGE-RELATED MACULAR DEGENERATION - A NEW APPROACH IN OPTICAL COHERENCE TOMOGRAPHY MONITORING AND QUANTITATIVE ASSESSMENT
}

\section{Elitsa G. Hristova, Zornitsa I. Zlatarova}

Department of Ophthalmology and Visual Science, Medical University Varna, Specialized Eye Hospital - Varna, Varna, Bulgaria

Corresponding Author:

Elitsa G. Hristova

Specialized Eye Hospital - Varna

15, Doyran Str.

Varna, 9002

Bulgaria

e-mail: elica gercheva@abv.bg

Received: October 30, 2014

Revision received: November 12, 2014

Accepted: December 19, 2014

\section{Summary}

The purpose of the study was to present the ability of Drusen analysis software tool to measure drusen area and volume in patients with dry age-related macular degeneration (AMD). Eleven patients with confirmed dry AMD aged 59-74 years were scanned with 3D OCT2000 Topcon 3D Macula scanning protocol by a single operator. All subjects underwent a complete ophthalmologic examination including best corrected visual acuity, indirect biomicroscopy, tonometry, fluorescein angiography and OCT. Drusen analysis was performed on the macula with $6.0 \times 6.0 \mathrm{~mm}$ volume cube scans and $512 \times 128$ pixels scan resolution. The results were presented along with calculated values in two clearly arranged reports. Mean follow-up period was 19 months (6-40). Count, area occupation, volume of the drusen and 3D retinal pigment epithelium (RPE) elevation map were presented in a Macula drusen analysis report. Drusen count and volume in 6 patients were increased at the end of follow-up period. There were 5 patients with regression in drusen count and area and volume of the drusen in 3 of them were higher than on previous examination. Another 2 were with regression not only in drusen count but also in their area and volume. With this software tool the status of RPE can be objectively and automatically examined in detail and can be followed up over time. OCT allows for precise quantitative evaluation and study of microstructural changes in patients with dry AMD and provides three-dimensional information of macular pathology in situ and in real time. This could be useful for determining stages and monitoring the progression of AMD.

Key words: optical coherence tomography, dry

\section{Introduction}

Age-related macular degeneration (AMD) is the leading cause of irreversible blindness among individuals older than 65 years in industrialized countries. It is an abnormality of the retinal pigment epithelium (RPE) that leads to photoreceptor degeneration of the overlying central retina, or macula, and loss of central vision $[1,2]$. The prevalence and incidence of AMD is likely to increase dramatically with the ageing of the population. 
It is estimated that approximately $30 \%$ of adults older than 75 years have some sign of AMD and that approximately $7 \%$ of these patients demonstrate advanced or late stages of the disease [3, 4]. In the United States 1.75 million people suffer from advanced AMD, and 7.3 million people are affected with intermediate AMD, which represents increased risk for development of advanced disease [5]. The same statistic in Europe shows 2.5 million people older than 65 years diagnosed with AMD and 1.1 million with visual impairments affected both eyes [6]. That is the reason for AMD to be considered a major public health problem with significant economic and social impact.

There are a number of classification schemes for AMD but the most commonly used one is The Age Related Eye Disease Study (AREDS) clinical classification [3]. The 4-stage classification of AMD from AREDS divides it into: No AMD, Early AMD, Intermediate AMD and Advanced AMD.

Early AMD is characterized by subretinal deposits, known as drusen, that measure greater than $60 \mu \mathrm{m}$ and hyper- or hypo-pigmentation of the RPE. Intermediate AMD is characterized by the accumulation of focal or diffuse drusen measuring greater than $125 \mu \mathrm{m}$ and hyper- or hypo-pigmentation of the RPE. Advanced AMD can be classified into either of two categories. The first of these is geographic atrophy (GA; i.e., dry, or non-exudative, AMD), which is characterized by a sharply delineated area of REP atrophy measuring at least $175 \mu \mathrm{m}$ along one dimension and including visible choroidal vessels. The alternative form of the advanced disease is choroidal neovascularization $(\mathrm{CNV}$; i.e., wet, or exudative, AMD), which may involve some or all of the following: subretinal neovascular membranes; subretinal fluid, exudates, and hemorrhages; pigment epithelial detachment (PED); and subretinal/intraretinal scarring [7].

The etiology and pathogenesis of AMD is likely multifactorial, involving a complex interaction of functional, metabolic, genetic and environmental factors, and remains poorly understood. These factors seems to create a stage for chronically developing changes in ocular structures of the macular region (photoreceptors, retinal pigment epithelium- RPE, Bruch's membrane, choriocapillaries), which may contribute to varying degrees of AMD. Some authors state that there are at least four processes contribute to the disease: lipofuscinogenesis (with its linkage to oxidative stress), drusogenesis, local inflammation and neovascularization (in the case of wet form) [812]. Several independent research groups have recently identified a common variant $(\mathrm{Y} 402 \mathrm{H})$ of the complement factor $\mathrm{H}(\mathrm{CFH})$ gene that may explain about $50 \%$ of AMD cases [13, 14]. This for the first time shows existence of a connection between a single gene anomaly and the pathogenesis of AMD.

Now available therapies are not causal treatments - they help to avoid further vision loss rather than to improve vision. Currently, there is no proven drug therapy that stops the progression of dry AMD. However, cessation of smoking and treatments based on AREDS [3] formula vitamin supplementation combined with a healthy diet are considered options for slowing disease progression. Most of the current therapies and new investigational treatments are directed at CNV.

In the past few years there has been an aggressive search for precise imaging methods to assess disease progression, which do not involve visual acuity. Probably, the best imaging strategy for complete assessment of dry AMD, which includes drusen shape, area, and volume, is the optical coherence tomography (OCT).

OCT is a non-invasive, high-resolution technique that produces in vivo cross-sectional images of tissues that resemble histological analysis. Spectral domain (SD) OCT, also known as Fourier domain OCT, makes it possible to differentiate several structural characteristics within the retina, including visualization of phototoreceptor and RPE layers, as well as subtle changes associated with the disease process [15]. SD-OCT also provides the opportunity to assess the effect of drusen on the geometry of the RPE in vivo and to identify drusen as localized elevations and disruptions in the RPE and Bruch's membrane complex [16].

The purpose of our study was to present the ability of Drusen analysis software tool of 3D OCT- 2000 to measure drusen area and volume in patients with dry age-related macular degeneration.

\section{Materials and Methods}

Eleven patients with confirmed dry AMD aged between 59-75 years were scanned with 3D OCT2000 [Topcon, Hasunuma-cho, Itabashi-ku, 
Tokyo, Japan] 3D Macula scanning protocol by a single operator. All subjects underwent a complete ophthalmic examination including best-corrected visual acuity, slit-lamp examination, Goldmann applanation tonometry, dilated stereoscopic fundus examination, fluorescein angiography and OCT. Exclusion criteria were refractive error $>6.00$ diopters sphere and/or 3.00 diopters cylinder, IOP $>21$ $\mathrm{mmHg}$, eyes with retinopathy due to any cause, eyes with any characteristic glaucomatous disc change. Drusen analysis was performed on the macula with $6.0 \times 6.0 \mathrm{~mm}$ volume cube scans and $512 \times 128$ pixels scan resolution, scan depth 2.3 $\mathrm{mm}$, horizontal resolution $20 \mu \mathrm{m}$, longitudinal resolution 5-6 $\mu \mathrm{m}$. The results were presented along with calculated values in two clearly arranged reports. All analysis was performed with SPSS software, version 19.0.

\section{Results}

Eleven eyes of 11 patients with dry AMD aged between 59 to 75 years $(66.73 \pm 7.01)$ were evaluated. Three of them were men $(27.3 \%)$ and 8 were women $(72.7 \%)$. Mean follow-up period was 19 months (range 6-40). Drusen count and volume in 6 patients were increased at the end of follow-up period. There were 5 patients with regression in drusen count, but area and volume of the drusen in 3 of them were higher than on a previous examination, other 2 were with regression not only in drusen count but also in their area and volume (Table 1).
Within Drusen analysis tool, there are two screens available for reviewing the results. The first screen shows count, area occupation, volume of the drusen and $3 \mathrm{D}$ retinal pigment epithelium (RPE) elevation map (Figure 1). The RPE elevation map is an en face image presented in a pseudo-colour map using a colour scale that highlights elevations. This map is presented as image overlaying the OCT fundus. The second one shows count, area occupation and drusen distribution in map centered on fovea and 9 standard ETDRS [17] regions (Figure 2).

Within 3D Macula scanning protocol shows OCT fundus image with the corresponding horizontal B-scan tomogram and ETDRS pseudo-colour macular map with the central subfield $1 \mathrm{~mm}$ in diameter and the inner and outer subfields with diameters of $3 \mathrm{~mm}$ and $6 \mathrm{~mm}$, respectively (Figure 3).

\section{Discussion}

Central vision is the vision we use to drive, read, recognize faces and perform daily tasks. At advanced stage of AMD central vision is so weak that faces can no longer be recognized and reading and writing are no longer possible.

Numerous risk factors have been reported for AMD but published evidence on some of them is insufficient or inconsistent. There are studies aimed to identify those risk factors with strong levels of evidence. Increasing age, current cigarette smoking, previous cataract surgery, and a family history of AMD has shown strong and

Table 1. Total drusen measurement in researched group

\begin{tabular}{lllllll}
\hline \multirow{2}{*}{$\begin{array}{l}\text { Patient } \\
\text { number }\end{array}$} & \multicolumn{2}{l}{ Drusen count } & Drusen area & \multicolumn{2}{l}{ Drusen volume } \\
\cline { 2 - 7 } & first visit & last visit & first visit & last visit & first visit & last visit \\
\hline 1. & 4 & 3 & 0.48 & 1.54 & 0.015 & 0.055 \\
\hline 2. & 7 & 10 & 0.82 & 1.19 & 0.024 & 0.037 \\
\hline 3. & 8 & 4 & 2.37 & 3.42 & 0.131 & 0.232 \\
\hline 4. & 6 & 7 & 0.38 & 0.49 & 0.011 & 0.015 \\
\hline 5. & 8 & 10 & 0.62 & 1.61 & 0.019 & 0.060 \\
\hline 6. & 11 & 10 & 1.10 & 1.30 & 0.046 & 0.058 \\
\hline 7. & 7 & 12 & 2.35 & 4.02 & 0.106 & 0.223 \\
\hline 8. & 17 & 12 & 1.99 & 1.45 & 0.111 & 0.055 \\
\hline 9. & 12 & 7 & 4.27 & 3.72 & 0.123 & 0.117 \\
\hline 10. & 13 & 15 & 3.45 & 3.80 & 0.193 & 0.210 \\
\hline 11. & 4 & 16 & 0.45 & 1.37 & 0.012 & 0.043 \\
\hline
\end{tabular}


Hristova E., Zlatarova Z. Dry age-related macular degeneration - a new approach...

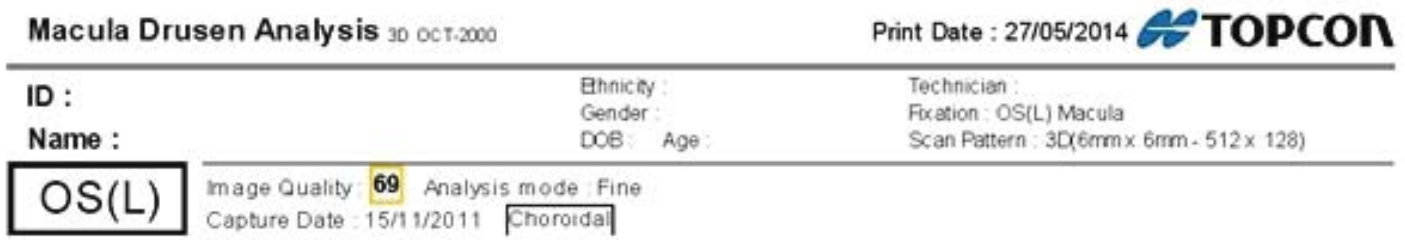
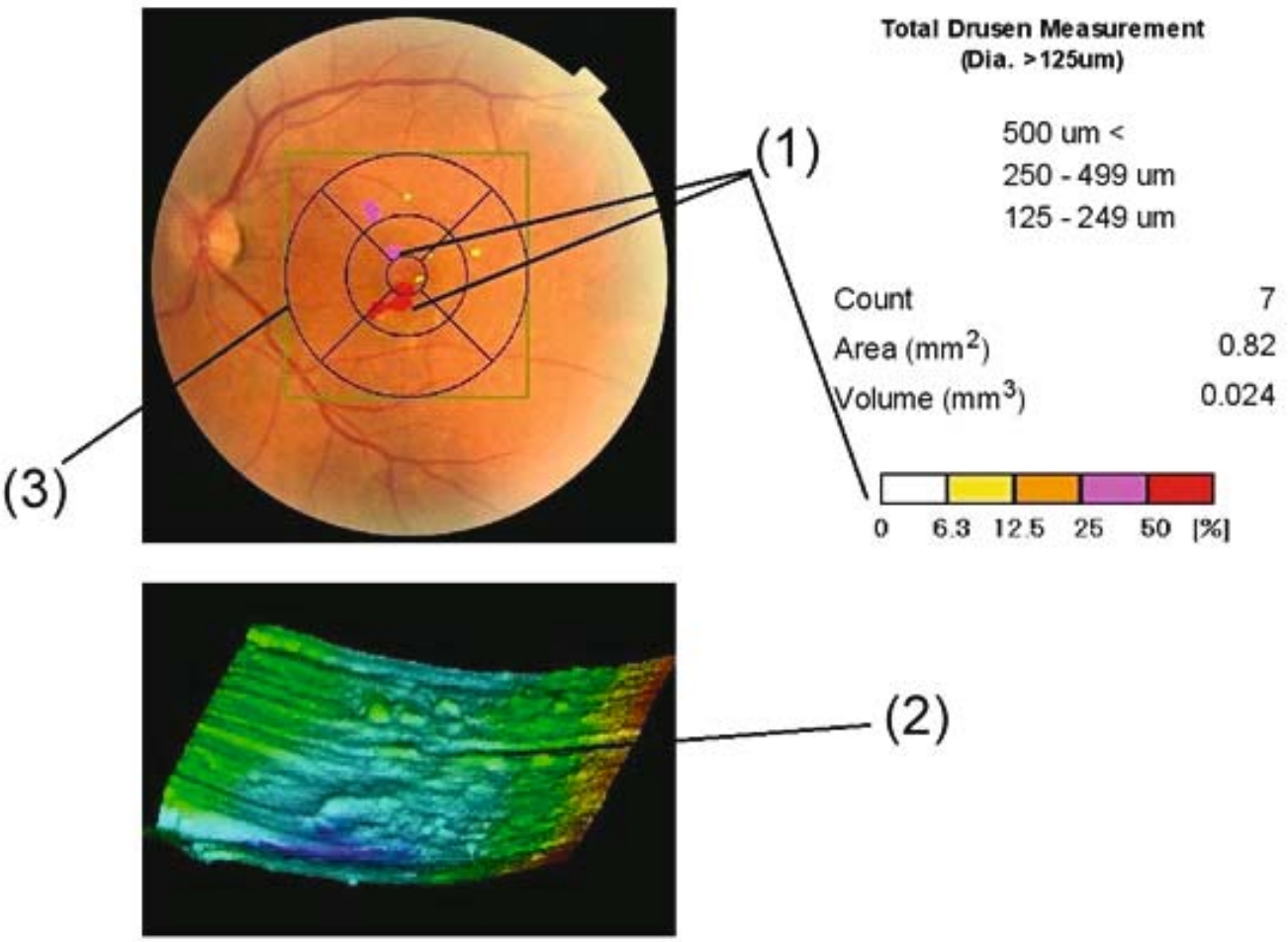

Figure 1. (1) Retinal pigment epithelium (RPE) elevation map overlaid on fundus image; (2) 3D RPE elevation map (3) Circles on the RPE elevation map centred on the fovea location with diameter $1 \mathrm{~mm}, 3 \mathrm{~mm}, 6 \mathrm{~mm}$
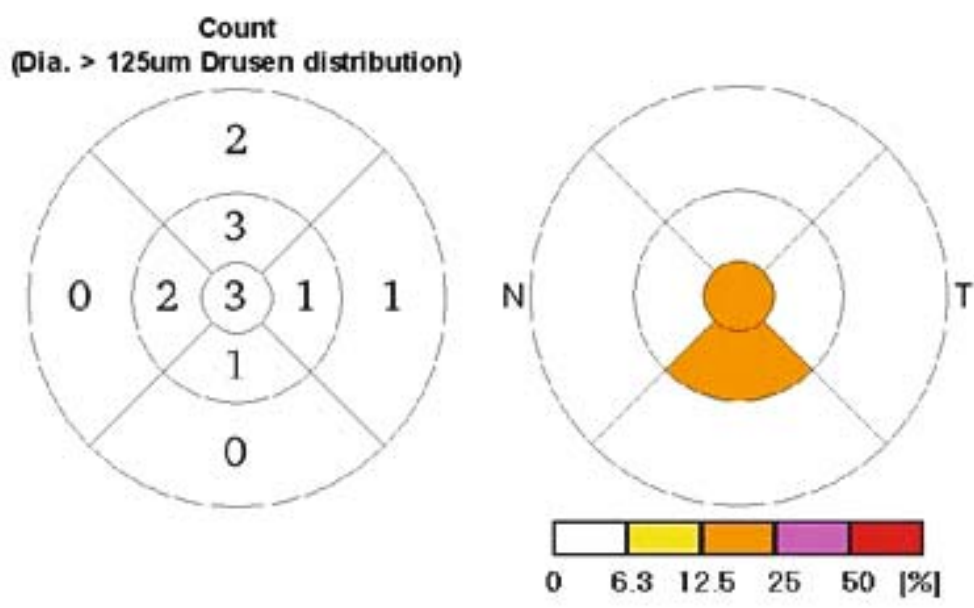

\section{(Dia. > 125um Drusen distribution)}

\section{Center}

inner-Nasal

inner-Superior

hner-Temporal

T inner-hferior

Outer-Nasal

Outer-Superior

Outer-Temporal

Outer-Inferior

Figure 2. Count, area occupation and drusen distribution in ETDRS [17] macular segmentation map 
Macula Analysis 30 oct-2000

ID :
Name :
OS(L)

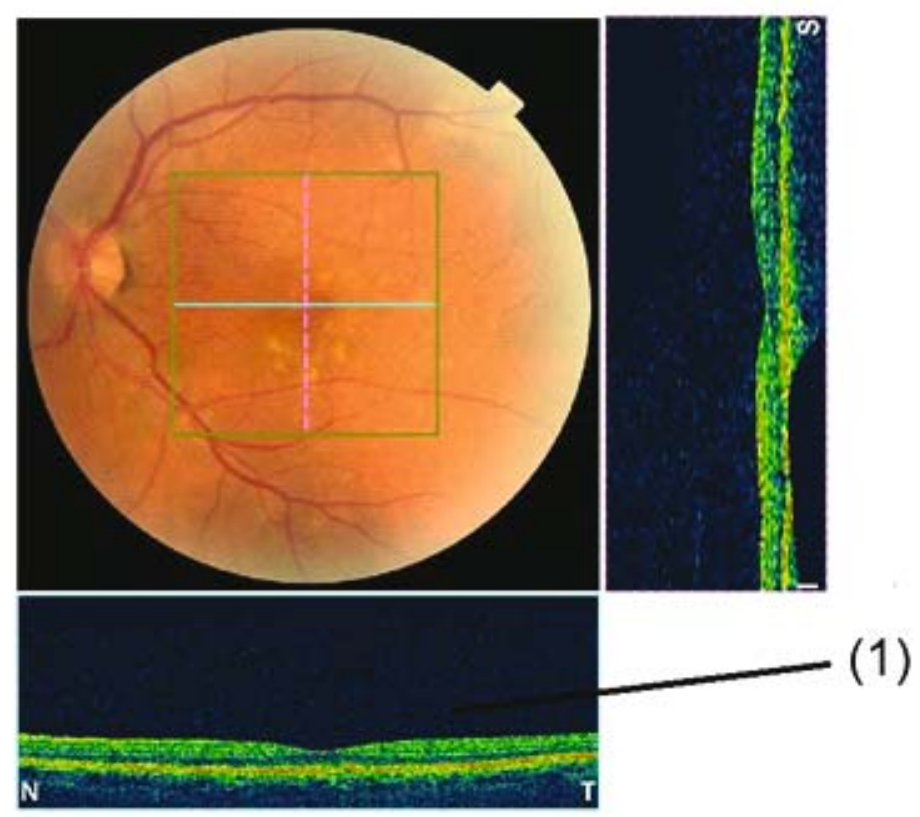

Image Quality 69 Analysis mode Fine

Image Quality 69 Analysis mode Fin
Capture Date : $15 / 111 / 2011$ Choroida
Print Date : 29108/2014 TOPCON

Technician

Fration: OS(L) Macula

Scan Pattern $30(6 \mathrm{~mm} \times 6 \mathrm{~mm}-512 \times 128)$

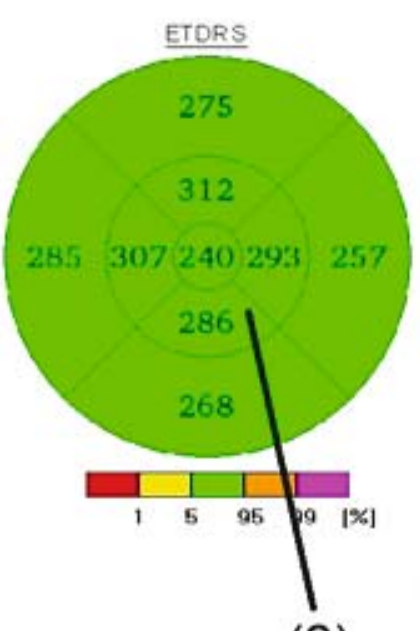

(2)

Figure 3. (1) Horizontal B-scan tomogram (2) Depiction of standard ETDRS map, showing macular thickness map centered on fovea and 9 standard ETDRS regions

consistent associations with late AMD. Risk factors with moderate and consistent associations included higher body mass index, history of cardiovascular disease and hypertension. Risk factors with weaker and inconsistent associations were gender, ethnicity, diabetes, iris colour, history of cerebrovascular disease, and serum total and HDL cholesterol and triglyceride levels [18]. Epidemiological studies have demonstrated differences in the prevalence of AMD based on ethnicity. In the second examination of Multiethnic Study of Atherosclerosis (MESA) prevalence of AMD in $4 \mathrm{racial} / \mathrm{ethnic}$ groups (white, black, Hispanic, and Chinese) were assessed. The results were $2.4 \%$ (black), $4.2 \%$ (Hispanic), 4.6\% (Chinese), to 5.4\% (white) [4]. White persons are generally more likely than black persons to have medium or large drusen, focal pigment abnormalities, and advanced AMD according to The Salisbury Eye Evaluation (SEE) Project with population-based sample of older black and white Americans [19].

Unlike investigating exudative AMD, where the natural history of vision loss is rapid and the resolution of the macular fluid can result in a profound visual acuity improvement, the natural history of vision loss in dry AMD is slow and at times imperceptible and there are no macular changes that have been shown to change rapidly with any therapy. Patients with dry AMD, even in the late atrophic stages, can maintain good central visual acuity until the disease progresses to the foveal center. As a result, it is unrealistic to expect early stage clinical trials to use visual acuity as a clinical endpoint.

The Drusen analysis software tool can reliably measure drusen morphology over time and this quantitative assessment of drusen could be used in both diagnosing and following up the patients and in various clinical trials. OCT uses a computerized algorithm to automatically measure drusen volume contained within circles with diameters of $3 \mathrm{~mm}$ and $6 \mathrm{~mm}$ and centered at the fovea. The natural history of drusen was studied using a similar algorithm and the average drusen volume was found to increase over time [20]. These observations suggest that drusen are morphologically dynamic with an undulating 
growth pattern in which drusen can go through repeated cycles of growth and shrinkage, with growth more likely than shrinkage [20]. These results are consistent with a model where drusen have a higher probability of growing rather than shrinking. Yehoshua et al. [1] demonstrated in their SD-OCT study that the likelihood of such a dramatic collapse in the drusen volume appeared to be dependent on drusen volume, with spontaneous drusen regression more commonly associated with larger baseline volumes. However, this event occurs over a range of drusen volumes and could not be predicted on the basis volume measurements or time of follow-up. Limitations of the present study include a nonstandardized follow-up interval for each patient. However, the behavior of drusen appeared to be more dependent on their baseline volume rather than on the length of follow-up.

One advantage of OCT images is that data obtained on prior and current visit are placed side by side and changes between visits are easily detectable and can be followed over time. Another major advantage of SD-OCT imaging is using only one type of scan for documenting both en face and cross-sectional images of the retina.

Gregori et al. quantitatively assessed drusen volume and area in 103 eyes from 74 patients with drusen and concluded that SD-OCT algorithm was highly reproducible for qualitative and quantitative assessment of drusen [21].

As drusen are a meaningful parameter of AMD, this tool may help to better evaluate disease progression. However, it has to be kept in mind, that very shallow drusen may be missed because of the threshold used by the algorithm or because of limitations in the sensitivity of the RPE segmentation This strategy will certainly be useful in assessing the natural history of nonexudative AMD and as a potential endpoint in testing the efficacy of new treatments for dry AMD.

\section{Conclusions}

The impact of developing AMD can be devastating to those who were independent and active prior to the onset of this impairment. There is no treatment for the dry form, but research is likely to produce some treatments in the coming years. OCT provides a solid base for such research with its three-dimensional information of macular pathology in situ and in real time in patients with dry AMD. This could be useful not only to determine stages and monitor the progression of AMD but to test the efficacy of new treatments for dry AMD as well.

\section{References}

1. Rattner, A. Nathans J. Macular Degeneration: Recent Advances and Therapeutic Opportunities. Nat Rev eurosci. 2006,7(11):860-72.

2. Haddad S, Chen CA, Santangelo SL, Seddon JM. The Genetics of Age-Related Macular Degeneration: A Review of Progress to Date. Surv Ophthalmol. 2006;51(4):316-63.

3. Age-Related Eye Disease Study Research Group. A Randomized, Placebo-Controlled, Clinical Trial of High-Dose Supplementation with Vitamins C and E, Beta Carotene, and Zinc for Age-Related Macular Degeneration and Vision Loss: AREDS Report No 8. Arch Ophthalmol. 2001; 119(10):1417-36.

4. Klein R, Klein BE, Linton KL. Prevalence of AgeRelated Maculopathy. The Beaver Dam Eye Study. Ophthalmology, 1992, 99(6), 933-43.

5. Friedman DS, O'Colmain BJ, Mucoz B, Tomany SC, McCarty C, de Jong PTVM, et al. Eye Diseases Prevalence Research Group. Prevalence of Age-Related Macular Degeneration in the United States. Arch Ophthalmol. 2004; 122(4):564-72.

6. Augood CA, Vingerling JR, de Jong PTVM, Chakravarthy U, Seland J, Soubrane G, et al. Prevalence of Age-Related Maculopathy in Older Europeans: The European Eye Study (EUREYE). Arch Ophthalmol. 2006;124(4):529-35.

7. Chen Y, Bedell M, Zhang K. Age-Related Macular Degeneration: Genetic and Environmental Factors of Disease. Mol Interv. 2010;10(5):271-81.

8. Campochiaro PA. Ocular Neovascularisation and Excessive Vascular Permeability. Expert Opin Biol Ther. 2004;4(9):1395-402.

9. Fine SL, Berger JW, Maguire MG, Ho AC. AgeRelated Macular Degeneration. N Engl J Med. 2000;342:483-92.

10. Kijlstra A, La Heij E, Hendrikse F. Immunological Factors in the Pathogenesis and Treatment of AgeRelated Macular Degeneration. Ocul Immunol Inflamm. 2005;13(1):3-11.

11. Klein R, Peto T, Bird A, Vannewkirk MR. The Epidemiology of Age-Related Macular Degeneration. Am J Ophthalmol. 2004; 137(3):486-95.

12. McConnell V, Silvestri G. Age-Related Macular Degeneration. Ulster Med J. 2005;74(2):82-92.

13. Bok, D. Evidence for an Inflammatory Process in Age-Related Macular Degeneration Gains New Support. Proc Natl Acad Sci U S A. 2005; 102(20):7053-4. 
14. Kuehn BM. Gene Discovery Provides Clues to Cause of Age-Related Macular Degeneration. JAMA. 2005;293(15):1841-5.

15. Loduca AL, Zhang C, Zelkha R, Shahidi M. Thickness Mapping of Retinal Layers by Spectral Domain Optical Coherence Tomography. Am J Ophthalmol. 2010;150(6);849-55.

16. Huang J, Liu X, Wu Z, Xiao H, Dustin L, Sadda S. Macular Thickness Measurements in Normal Eyes with Time Domain and Fourier Domain Optical Coherence Tomography. Retina. 2009;29(7):9807.

17. Early Treatment Diabetic Retinopathy Study Design and Baseline Patient Characteristics. ETDRS Report Number 7. Ophthalmology. 1991;98(5 Suppl):741-56.

18. Chakravarthy U, Wong TY, Fletcher A, Piault E, Evans C, Zlateva G, et al. Clinical Risk Factors for Age-Related Macular Degeneration: A Systematic Review and Meta-Analysis. BMC Ophthalmol. 2010;10:31.
19. Bressler SB, Mucoz B, Solomon SD, West SK, Salisbury Eye Evaluation (SEE) Study Team. Racial Differences in the Prevalence of AgeRelated Macular Degeneration: The Salisbury Eye Evaluation (SEE) Project. Arch. Ophthalmol. 2008;126(2):241-5.

20. Yehoshua Z, Wang F, Rosenfeld PJ, Penha FM, Feuer WJ, Gregori G. Natural History of Drusen Morphology in Age-Related Macular Degeneration Using Spectral Domain Optical Coherence Tomography. Ophthalmology. 2011;118(12):2434-41.

21. Gregori G, Wang F, Rosenfeld PJ, Yehoshua Z, Gregori NZ, Lujan BJ, et al. Spectral Domain Optical Coherence Tomography Imaging of Drusen in Nonexudative Age-Related Macular Degeneration. Ophthalmology. 2011 ; 118(7):1373-9. 